\title{
Ets-1 drives breast cancer cell angiogenic potential and interactions between breast cancer and endothelial cells
}

\author{
ALESSANDRO FURLAN ${ }^{1,2}$, CHANTAL VERCAMER $^{1}$, LAURENT HELIOT ${ }^{2}$, \\ NICOLAS WERNERT ${ }^{3}$, XAVIER DESBIENS ${ }^{1}$ and ALBIN POURTIER ${ }^{1}$ \\ ${ }^{1}$ UMR8161 CNRS/University of Lille/Pasteur Institute of Lille, Biology Institute of Lille, \\ F-59021 Lille CEDEX; ${ }^{2}$ UMR8523 PhLAM, CNRS/University of Lille, \\ 59655 Villeneuve d'Ascq CEDEX, France; ${ }^{3}$ Institute of Pathology, \\ University of Bonn, 53011 Bonn, Germany
}

Received April 17, 2018; Accepted September 10, 2018

DOI: 10.3892/ijo.2018.4605

\begin{abstract}
Ets-1 transcription factor overexpression in breast cancers is associated with invasive features and is associated with a poor prognosis. Beyond its role in driving carcinoma cell invasion, in this study, we wished to determine whether Ets-1 overexpression in cancer cells promotes angiogenesis by creating a paracrine pro-invasive environment for endothelial cells as well. To address this question, we set up different co-culture models of cancer cells with endothelial cells. Conditioned media from cancer cells induced endothelial cell proliferation, migration and morphogenesis in matrix models. Of note, co-culture assays in three-dimensional matrix models also revealed the reciprocal induction of cancer cell morphogenesis by endothelial cells, in support of an angiocrine action on tumor cells. Ets-1 emerged as a key regulator of the angiogenic potential of breast cancer cells, favoring their ability to induce, in a paracrine manner, the morphogenesis of endothelial cells and also to physically interact with the latter. Nevertheless, Ets-1 overexpression in cancer cells also restrained their chemoattractive potential for endothelial cells both in Boyden chambers and in ex vivo 3D co-cultures. Finally, Ets-1 modulation in breast cancer cells qualitatively altered the angiogenic pattern of experimental in vivo tumors, with a balance between vessel recruitment and intratumoral small capillaries sprouting. Taken together, our data highlight a critical and intriguing role for Ets-1 in the angiogenic potential of breast cancer cells, and reveal another facet of Ets-1 oncogenic activities.
\end{abstract}

Correspondence to: Dr Alessandro Furlan or Dr Albin Pourtier, UMR8161 CNRS/Université de Lille/Institut Pasteur de Lille, Institut de Biologie de Lille, BP 447, 1 rue du Pr Calmette, F-59021 Lille CEDEX, France

E-mail: alessandro.furlan@univ-lille.fr

E-mail: albin.pourtier@univ-lille.fr

Key words: breast cancer, angiogenesis, Ets-1, transcription factor, cell interactions, 3D matrix models, co-culture

\section{Introduction}

Despite improvements in patient care over the past years, breast cancer remains a leading cause of mortality among women worldwide, accounting for $>500000$ deaths in 2012 (1). Breast tumors are most often angiogenic, i.e., they induce the development of neo-vessels to their profit. Angiogenesis is associated with lymph node metastasis and a poor prognosis for breast cancer patients (2). Anti-angiogenic agents have thus emerged as promising therapies (3). Nevertheless, such treatments have thus far failed to significantly improve the overall survival of breast cancer patients (4). A better knowledge of the mechanisms underpinning the complex interactions between cancers and vessels may aid in resolving this issue and in improving the therapeutic strategies.

Sprouting angiogenesis occurs through a branching morphogenesis process and requires endothelial cell proliferation, migration and maturation to form new capillaries. The latter step of differentiation is often lacking during tumoral angiogenesis, which leads to more permeant vessels that are hardly effective, but may facilitate the access of tumor cells to the systemic circulation (5). A better understanding of the mechanisms that induce cancer cells to trigger the angiogenic response and modulate the vessel features should provide crucial information with which to target this critical phenomenon in cancer progression.

Ets-1 proto-oncogene overexpression in breast cancers is associated with a poor prognosis (6). Ets-1 is a critical regulator of invasion induced by dialogues between the epithelium and mesenchyme (7). We previously demonstrated that Ets-1 overexpression stimulated breast cancer cell scattering through the coordination of several classes of invasion-related molecules (8). Among these, Ets-1 regulates several soluble factors potentially involved in angiogenesis, such as growth factors or extracellular matrix proteases, which prompted us to determine whether Ets-1 overexpression in breast cancer cells can trigger a paracrine angiogenic environment.

To address this issue, we manipulated Ets-1 activity in cancer cells, either by overexpressing Ets-1 or by expressing a dominant negative mutant, as previously described $(8,9)$. In this study, we investigated the effects of such manipulations 
upon the interactions between cancer and endothelial cells. We performed direct heterotypic co-cultures of breast cancer cells with normal endothelial cells, or administered conditioned media collected from one cell type on the other. We used three-dimensional matrix models, which recreate a physiological environment (10), which allowed us to mimic several physiological aspects of capillary formation. Finally, we examined the effects of Ets-1 on the angiogenic pattern of experimental tumors in vivo. This allowed us to highlight an interesting and complex role of Ets-1 expression by breast cancer cells in controlling the angiogenic induction.

\section{Materials and methods}

Cell culture. Mouse mammary tumor epithelial cells (MMT CCL-51 cells; ATCC, Manassas, VA, USA) were routinely cultured in DMEM (BioWhittaker; Lonza Walkersville, Inc., Walkersville, MD, USA) supplemented with penicillin, streptomycin, non-essential amino acids, L-glutamine and $10 \%$ fetal calf serum (Gibco/Thermo Fisher Scientific, Waltham, MA, USA). They were infected in parallel by supernatants from virus-producing GP+E86 cells (kind gift from Dr Arthur Bank, Columbia University, New York, NY, USA) transfected with MFG retroviral constructs to overexpress Ets-1 or a dominant negative mutant composed of the DNA binding domain of Ets-1, as previously described $(8,9)$. Briefly, the cDNA sequence encoding either the full sequence or the DNA-binding domain of murine Ets1 was cloned upstream of the internal ribosome entry site (IRES) and the neomycinresistance gene then inserted into a retroviral MFG vector in replacement of the Gag, Pol and Env genes, as previously described in detail (11).

Murine endothelial mouse spleen stroma (MSS-31) cells were obtained from Dr N. Yanai (12). They were grown in $\alpha$-MEM (BioWhittaker; Lonza Walkersville, Inc.) supplemented with penicillin-streptomycin, glutamine and $10 \%$ fetal calf serum.

All cell lines used were checked with the list of known misidentified cell lines available from the International Cell Line Authentication Committee and ExPASy Cellosaurus, and they are not misidentified nor contaminated.

Co-culture models. For two-dimensional and three-dimensional matrix models, we used a matrix substrate composed of type I collagen and Matrigel ${ }^{\mathrm{TM}}$, as previously described $(8,9)$. Conditioned media were prepared by respectively seeding $3 \times 10^{6}$ MMT or $1 \times 10^{6}$ MSS-31 cells suspended in $3 \mathrm{ml}$ appropriate medium upon $100 \mathrm{~mm}$-diameter Petri dishes coated with this matrix. Twenty-four hours later, the cell culture supernatants were harvested, filtered through Millex $0.22 \mu \mathrm{m}$ filters, and stored at $-80^{\circ} \mathrm{C}$ in Sorenson ${ }^{\mathrm{TM}}$ pre-lubricated tubes prior to their use.

For two-dimensional MSS-31 morphogenesis assays, $150 \mu \mathrm{l}$ of matrix was poured in 48 -well plates and incubated for $30 \mathrm{~min}$ at $37^{\circ} \mathrm{C}$. The MSS-31 cells were then seeded at a density of 20,000 cells per well, in 5\% FCS $\alpha$-MEM. An equal volume of conditioned medium from the MMT cells, or DMEM as a control, was then added. The media were refreshed every other day. At the end of the experiment, cellular structures were stained with neutral red $(0.1 \%$ in PBS) then fixed with $4 \%$ paraformaldehyde in PBS. Images were acquired using a Leica DMIRB inverted microscope (Leica Microsystems GmbH, Wetzlar, Germany).

For three-dimensional MSS-31 morphogenesis assays, a first acellular layer of matrix was deposited in 48-well plates. The MSS-31 cells were then resuspended in that matrix solution and seeded at a density of 25,000 cells per well. Serum-free conditioned media from the MMT cells or DMEM were added and refreshed every 3 days. Images were acquired under a phase contrast light or epifluorescence using a Leica DMIRB inverted microscope (Leica Microsystems $\mathrm{GmbH}$ )

For direct co-culture assays, the MMT and MSS-31 cells were respectively labeled by diI and diO Vybrant ${ }^{\mathrm{TM}}$ Cell-Labeling solutions (V-22885 and V-22886; Molecular Probes/Thermo Fisher Scientific), according to the manufacturer's instructions.

For three-dimensional co-cultures, 4,000 MMT cells suspended in $300 \mu \mathrm{l}$ of matrix were seeded in 24-well plates above a first acellular layer matrix. A suspension of 2,000 MSS-31 cells was then added upon these gels.

Image analysis. Primary branch thickness, fluorescence intensity and capillary lumen areas were quantified with ImageJ measurement tools (National Institutes of Health, Bethesda, Maryland, USA, https://imagej.nih.gov/ij/).

Cell proliferation. The MSS-31 cells were seeded in 96-well plates at a density of 500 cells per well, and $30 \%$ of their culture medium was replaced by conditioned media from MMT cells, or complete DMEM as a control. Reciprocally, the MMT cells were seeded at a density of 2,000 cells per well in 12 -well plates, and $30 \%$ of their culture medium was replaced by conditioned media from MSS-31 cells or complete $\alpha$-MEM as a control. The media were refreshed every day. The cell number was assessed by MTT assay (M-2128; Sigma-Aldrich, St. Louis, MO, USA). Briefly, the cells were incubated at $37^{\circ} \mathrm{C}$ for $2 \mathrm{~h}$ in presence of $1 \mathrm{mM}$ MTT, and the purple formazan crystals were then dissolved in a solution of $5 \mathrm{mM} \mathrm{HCl}$ in isopropanol. The plates were shaken for $5 \mathrm{~min}$ and the optical density was then measured at a wavelength of $570 \mathrm{~nm}$ with an MRX Revelation plate reader (Dynex Technologies GmBH, Denkendorf, Germany).

Wound assays. The MMT cells were seeded in $60 \mathrm{~mm}$ diameter Petri dishes and grown until sub-confluence. A wound was created on the cell layer using a scraper. The dishes were rinsed 3 times with fresh medium, and equal volumes of DMEM and conditioned medium from the MSS-31 cells, or 5\% FCS $\alpha$-MEM as a control, were then added. Cell migration in the denuded area was recorded $24 \mathrm{~h}$ later. Images were acquired using a Leica DMIRB inverted microscope (Leica Microsystems GmbH).

Transwell assays. The MMT cells were seeded at a density of 10,000 cells per well in 24-well Falcon ${ }^{\mathrm{TM}}$ Companion plates (Thermo Fisher Scientific). Twenty hours later, the medium culture was refreshed and 15,000 MSS-31 cells per well were seeded under serum-free conditions upon Transwell tissue culture inserts with $8.0 \mu \mathrm{m}$ pore size (Falcon ${ }^{\mathrm{TM}}$ ), at a density of 25,000 cells per well. Alternately, MSS-31 cells were seeded at a density of 20,000 cells per well in 24-well Companion plates $\left(\right.$ Falcon $\left.^{\mathrm{TM}}\right)$. Twenty hours later, the medium was replaced and 
MMT cells were seeded in Transwell tissue culture filters with $8.0 \mu \mathrm{m}$ pore size $\left(\right.$ Falcon $\left.^{\mathrm{TM}}\right)$, at a density of 25,000 cells per well. The cells were allowed to migrate for $30 \mathrm{~h}$, then non-migratory cells were removed from the top of the filter by swiping with humidified cotton swabs. Cells that had migrated through the filter pores to the lower face of the inserts were fixed in $4 \%$ paraformaldehyde in PBS, their nuclei were stained with Hoechst at $5 \mathrm{mg} / \mathrm{l}$ in PBS, and counted. Values from the control conditions were set as the reference value of 1 . Data are expressed as means of 3 independent experiments.

Cell adhesion. The MMT cells were fluorescently labeled as described above in 'Co-culture models' section, and seeded in 12-well plates at a density of 50,000 cells per well, upon MSS-31 cell confluent monolayers. Following $45 \mathrm{~min}$ of incubation at $37^{\circ} \mathrm{C}$, wells were thoroughly rinsed 3 times by fresh medium, and the plates were further incubated for 4 additional hours at $37^{\circ} \mathrm{C}$ so that the cells could complete their adhesion. The cells were then fixed with paraformaldehyde and observed under epifluorescence to count the fluorescent MMT cells, using a Leica DMIRB inverted microscope (Leica Microsystems $\mathrm{GmbH})$. Values from the control conditions were set as the reference value of 1 . The results are means of 3 independent experiments.

Zymography. Conditioned media or membrane-enriched fractions were analyzed for MMP-2 and MMP-9 gelatinolytic activities. Briefly, SDS-PAGE was performed under non-reducing conditions in $7.5 \%$ polycrylamide gels containing $0.1 \%$ gelatin. The gels were then rinsed for $30 \mathrm{~min}$ in $10 \mathrm{mM}$ Tris- $\mathrm{HCl} / 2.5 \%$ Triton $\mathrm{X}-100(\mathrm{pH} 8.0)$, and further incubated at $37^{\circ} \mathrm{C}$ for $20 \mathrm{~h}$ in $50 \mathrm{mM}$ Tris- $\mathrm{HCl}, 0.5 \mathrm{mM} \mathrm{CaCl}_{2}, 1 \mu \mathrm{M} \mathrm{ZnCl}_{2}$ $(\mathrm{pH} 8.0)$ to restore gelatinases activity. Gels were stained in $0.25 \%$ Coomassie blue R250 and destained in 7\% acetic acid plus $25 \%$ methanol. Gelatin degradation is detected by the appearance of translucent bands in the dark blue-stained gelatin background.

Western blot analysis. The cells were subjected to hypotonic and thermic lysis as previously described (8). Protein amounts were measured according to the Bradford method (13). Proteins from membrane-enriched fractions or cell conditioned media were separated by polyacrylamide gel electrophoresis, then transferred onto nitrocellulose membranes. Blocking was performed with 5\% non-fat dry milk in PBS Tween. Primary anti-VE-cadherin antibody was purchased from Bender MedSystems $^{\mathrm{TM}}$ (BMS158) and used at $1 \mu \mathrm{g} / \mathrm{ml}$. For the loading control, the primary anti-GAPDH (6C5) sc-32233 was purchased from Santa Cruz Biotechnologies (Santa Cruz, CA, USA) and used at used at $0.1 \mu \mathrm{g} / \mathrm{ml}$. Secondary peroxidaseconjugated antibodies (anti-mouse 115-035-146 for GAPDH, and anti-rabbit 711-035-152 for VE-cadherin) were purchased from Jackson ImmunoResearch Laboratories, Inc (West Grove, PA USA) and used at $0.08 \mu \mathrm{g} / \mathrm{ml}$. Peroxidase activity was recorded by chemoluminescent detection using the ECL $^{\mathrm{TM}}$ kit and Hyperfilm ${ }^{\mathrm{TM}} \mathrm{MP}$ from Amersham Biosciences/ GE Healthcare (Little Chalfont, UK).

In vivo experiments. In vivo experiments were performed according to approved institutional guidelines. Specific authorization no. 59-00994 was granted by the institutional veterinary authorities.

Subcutaneous injections. MMT cells were subcutaneously injected into female nu/nu BALB/c mice, in Growth FactorReduced Matrigel ${ }^{\mathrm{TM}}$, at a density of 300,000 cells per $100 \mu \mathrm{l}$ plug. Mice were housed in a sterile atmosphere in isolators $\left(25^{\circ} \mathrm{C}\right.$; humidity range maintained between 40 and $\left.70 \%\right)$ with food and water ad libitum, and 8-week-old mice, weighing approximately $20 \mathrm{~g}$, were used in the experiments. A total of 4-5 mice were used for each condition. Ten days later, the anesthetized animals received injections of $100 \mathrm{ml}$ PBS with 2\% FITC-dextran (MW 250 000, Sigma-Aldrich, St. Louis, MO, USA) in the tail vein. An excision was then performed in the skin of the flanks to unveil the underlying tumor vasculature. Mice were then sacrificed and the tumors were fixed in PBS with 4\% paraformaldehyde and processed for paraffin embedding.

Ex vivo tumor explant angiogenesis assay. Tumors obtained in the above-mentioned experiment were partially cut in small sections prior to the fixation step. Fragments of equivalent size were seeded upon matrix gels in which 10,000 diI-labelled MSS-31 cells had been embedded.

Metastasis assays. For lung colonization assays, the MMT cells were injected in the tail vein of female nu/nu BALB/c mice $(100,000$ cells per mouse in sterile PBS). Four mice, different from those used for subcutaneous injections, were used for each condition. The mice were sacrificed 12 days later and tumors were dissected. The lungs were fixed with 4\% paraformaldehyde in PBS and paraffin-embedded.

Immunohistology. Immunohistodetection of the endothelial markers was performed as previously described (14). Briefly, $7-\mu \mathrm{m}$-thick tissue sections were dewaxed and incubated for $30 \mathrm{~min}$ in PBS with $2.5 \mathrm{mg} / \mathrm{ml}$ trypsin to restore antigen accessibility. Following endogenous peroxidase inhibition, blocking was carried out with $0.5 \%$ casein in PBS. The sections were incubated overnight at $4^{\circ} \mathrm{C}$ with anti-CD31/PECAM (01951 A, $50 \mu \mathrm{g} / \mathrm{ml})$ or anti-CD34 (553731, $50 \mu \mathrm{g} / \mathrm{ml})$ antibodies (Pharmingen/BD Biosciences, San Jose, CA, USA), then sequentially incubated with biotinylated anti-rat IgG secondary antibodies (65-153, Jackson ImmunoResearch Laboratories) diluted 1:5,000 and avidin peroxidase (A-2004, Vector Laboratories, Burlingame, CA, USA) diluted 1:1,000. Peroxidase activity was revealed by DAB chromogen (Dako, Glostrup, Denmark) and tissues were counterstained with Mayer's hematoxylin.

Statistical analysis. Each experiment was repeated at least 3 times and the results are expressed as the mean \pm standard error. Statistical analysis was carried out with RStudio (RStudio, Inc., Boston, MA, USA) open source data analysis software. ANOVA with the post hoc Tukey range test provided adjusted P-values. Statistical significance was reached with values of $\mathrm{P}<0.05$, or $\mathrm{P}<0.01$; otherwise differences were declared non-significant (NS).

\section{Results}

Breast cancer cells and endothelial cells establish a bidirectional dialogue in co-culture models. In order to examine angiogenic induction by breast cancer cells, we 
A

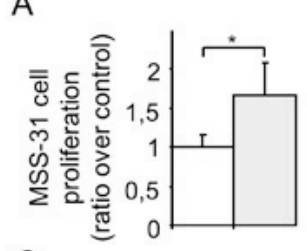

c
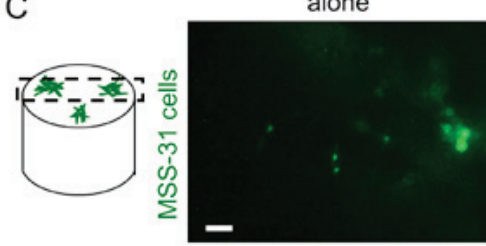

$\mathrm{D}$

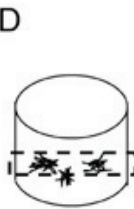

alone
B

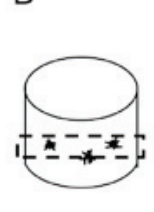

alone

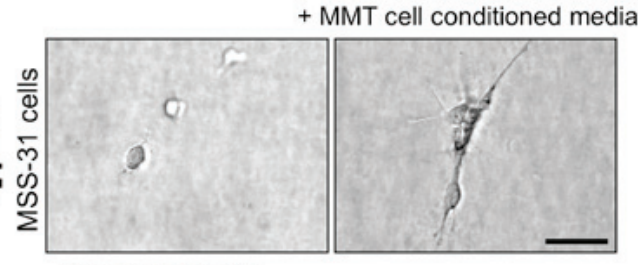

above MMT cells

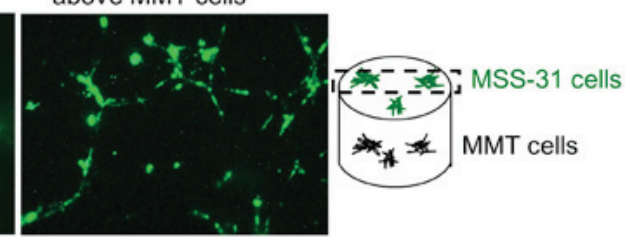

under MSS-31 cells

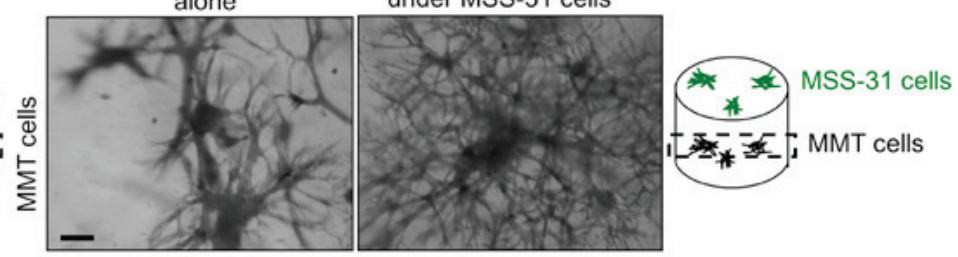

phase contrast

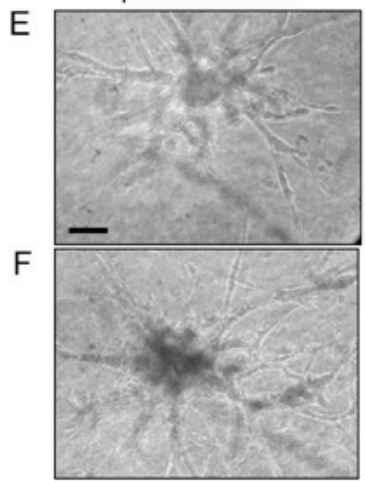

MMT cells
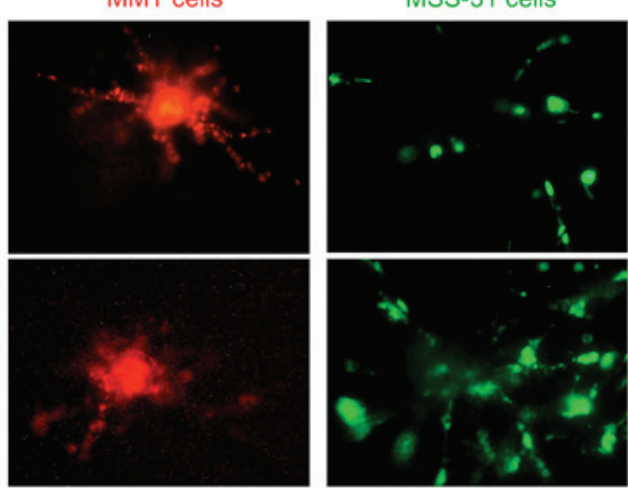

Figure 1. Breast cancer cells and endothelial cells establish a bidirectional dialogue in co-culture models. (A) Endothelial MSS-31 cell proliferation was measured after a 3-day culture in $70 \%$ complete medium $+30 \%$ MMT neo cancer cell conditioned media (gray bar) or corresponding vehicle (white bar); $n=3$; ${ }^{*} \mathrm{P}<0.05$. (B) MSS-31 cells were cultured in serum-free 3D matrix gels in absence or presence of MMT neo cell conditioned media. Images were acquired after a 10-day culture. (C) Fluorescent diO-labeled endothelial cells were cultured upon a 3D matrix gel containing or not cancer cells for 5 days. (D) MMT neo cancer cells were embedded within the 3D matrix gel, in absence or presence of endothelial MSS-31 cells on top of the gels. Images were acquired at the level of cancer cells in the gel. (E and F) DiI-labeled MMT cells (red fluorescence) form connected structures with diO-labeled MSS-31 cells (green fluorescence) in 3D co-cultures, observed at (E) 3 days or (F) 8 days after cell seeding. Labeling weakening over time does not allow a proper and unambiguous identification of cell type at heterotypic contact sites. Scale bars, $50 \mu \mathrm{m}$.

set up co-culture models of MMT cancer cells with normal endothelial MSS-31 cells. We first collected conditioned media from MMT control cancer cells, and administered them to endothelial cell cultures. Endothelial cells were responsive to cancer cell soluble stimuli and displayed an increased proliferation (Fig. 1A), when compared to the condition control in which they received the corresponding vehicle alone. To more closely mimic physiological angiogenic induction, we placed the endothelial cells in 3D matrix gels composed by type I collagen and reconstituted basal membrane-like compounds, namely Matrigel ${ }^{\mathrm{TM}}$. In the absence of additional stimuli, endothelial cells failed to divide in this environment. On the contrary, when they received conditioned media from the cancer cells, the endothelial cells began to grow, elongate and organize (Fig. 1B).

Finally, we took our experiment a step further and co-cultured both the cancer and endothelial cells in such matrix gels. We used the intravital fluorochromes, diI and diO, to label each cell type prior to its seeding. Endothelial cells were grown on top of the gels containing (or not) cancer cells. Within 1 week, the endothelial cells formed a layer under the control conditions, and became organized as a network when the cancer cells were cultured in the gels (Fig. 1C, highlighting diO-labeled endothelial cells). At the same time, the MMT cancer cells grew within the gel and lost most of their fluorescent signal during cell divisions. The presence of endothelial cells upon the gel stimulated the morphogenesis of underlying cancer cells, with more branching points and thinner primary branches when compared to the control condition (Fig. 1D), thus providing evidence of a reciprocal dialogue between cancer and endothelial cells in co-culture.

When the MMT cancer cells grew in the vicinity of the gel surface, it was possible to observe the formation of connected branched structures between cancer and endothelial cells (Fig. 1E and F). These hybrid structures developed over time ( 8 days of co-culture for Fig. 1F vs. 3 days for Fig. 1E); however, cell fluorescence weakening did not allow an unambiguous observation of heterotypic cell contacts. The increased 
A
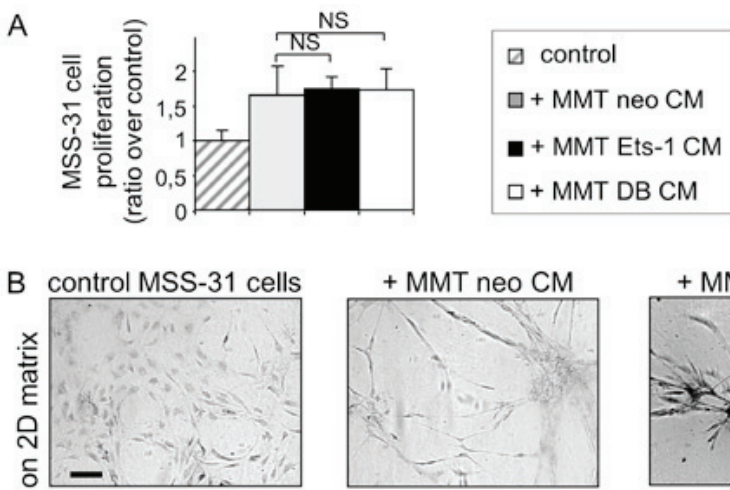

+ MMT neo CM

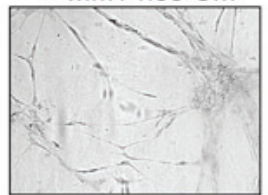

+ MMT Ets-1 CM
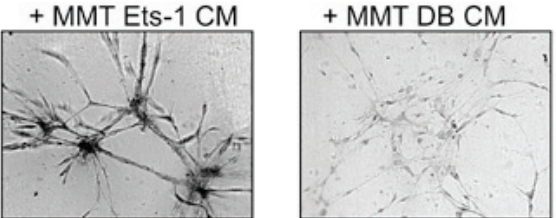

control MSS-31 cells + MMT neo CM

+ MMT Ets-1 CM

+ MMT DB CM
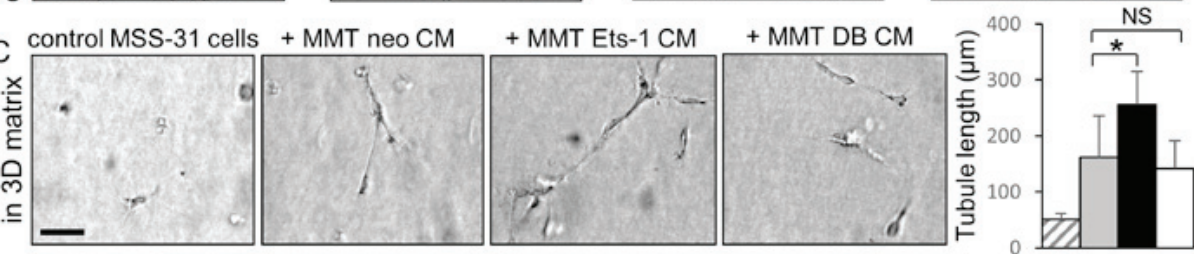

D MSS-31 cells alone

over MMT neo
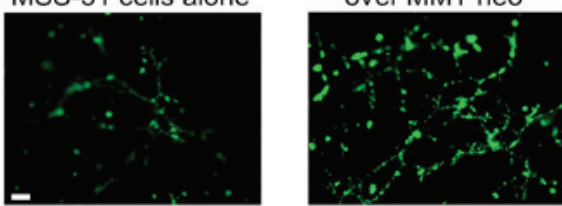

over MMT Ets-1

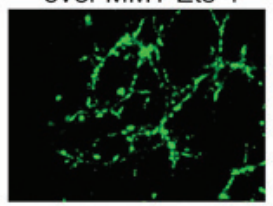

over MMT DB

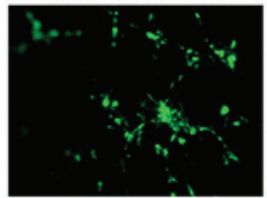

E at the beginning after co-culture

$\mathrm{F}$

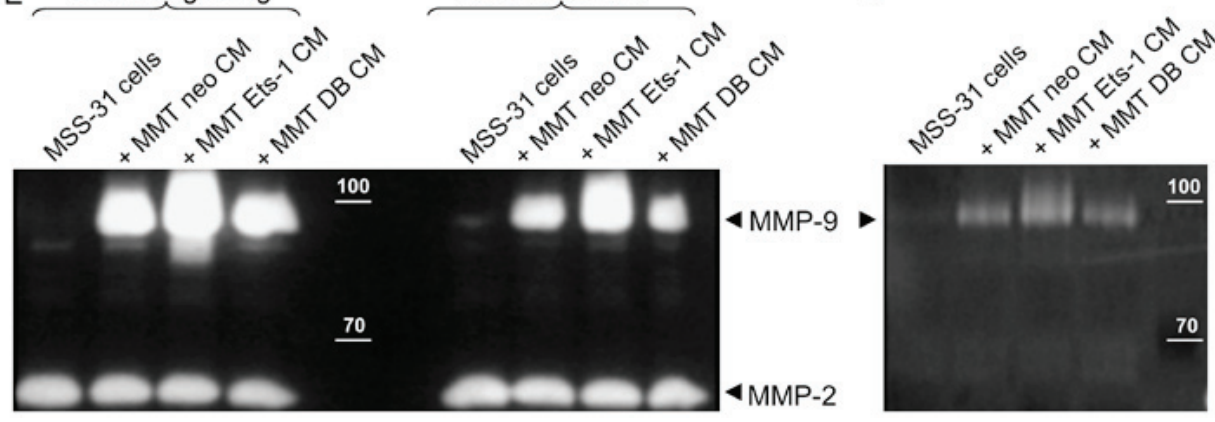

Figure 2. Ets-1 enhances breast cancer cell ability to induce endothelial cell morphogenesis but not proliferation. (A) MSS-31 cell proliferation was measured after a 3-day culture in 70\% complete medium + 30\% MMT cancer cell conditioned media or corresponding vehicle. Values are representative of 3 independent experiments. NS, non-significant. (B) MSS-31 cell morphogenesis on top of matrix gels is increased in response to MMT cell conditioned media, in an Ets-1-dependent manner. Images were acquired after neutral red staining of a 6-day culture. (C) MMT cell conditioned media enhance MSS-31 cell morphogenesis within 3D matrix gels in serum-free conditions, in an Ets-1-dependent manner. Images were acquired after a 10-day culture. "P<0.05; NS, non-significant (D) DiO-labeled MSS-31 cells were cultured upon matrix gels containing MMT cells. Endothelial cell networks were visualized under epifluorescence. (E) Gelatin zymography was performed with MSS-31 culture supernatants, just after (left columns) or $24 \mathrm{~h}$ after (right columns) the addition of conditioned media from MMT neo, MMT Ets-1 or MMT DB cells, or their control non-conditioned medium. MMP-2 and MMP-9 activities are detected as translucent bands amid the blue-stained gelatin substrate, and can be discriminated thanks to their different molecular weight. The position of 100 and $70 \mathrm{kDa}$ known from the molecular ladder is indicated by the white dashes. Zymography evidences the decrease in MMP-9 activity in supernatants after incubation with endothelial cells. (F) Gelatin zymography of MSS-31 cell membrane extracts following a 5-min incubation with conditioned media from MMT cells, or their medium in control, highlights the recruitment of cancer-derived MMP-9 to the endothelial cell membrane. Scale bars, $50 \mu \mathrm{m}$.

presence of endothelial cells after 8 days of co-culture suggests a possible recruitment of endothelial cells by cancer cells, although we cannot completely rule out the contribution of endothelial cell proliferation.

Ets-1 enhances breast cancer cell ability to induce endothelial cell capillary-like morphogenesis, but not proliferation. Ets-1 regulates the expression of invasion-related molecules by breast cancer cells. In order to determine whether its expression by cancer cells can influence their ability to induce angiogenesis, the MMT cells were infected by MFG retroviral vectors to overexpress Ets-1 or the dominant negative mutant DB (for DNA binding domain), as previously described (8).
These cells will be respectively termed MMT Ets-1 and MMT DB cells hereinafter. MMT cells infected with the empty vector carrying neomycin resistance were used as control MMT neo cells. The validation of construct expression has been previously provided (8).

To assess the impact of these manipulations on cancer cell angiogenic ability, MSS-31 murine endothelial cells received conditioned media (CM) from our different MMT sublines. The administration of these conditioned media for 3 days stimulated MSS-31 cell proliferation (+67\% with MMT neo CM, Fig. 2A). Neither Ets-1 overexpression nor the dominant negative expression statistically altered this induced proliferation $(+75 \%$ with MMT Ets- 1 CM, and +74\% with MMT DB CM, Fig. 2A; NS). 
Proliferation is required for angiogenesis, but invasion and cell organization represent other key features of this process. Endothelial cell branching morphogenesis in matrix gels recapitulates several aspects of physiological capillary formation (15). In this study, we thus investigated the role of Ets-1 in MMT cell angiogenic potential in matrix models. When cultured upon these matrix gels in the absence of proper stimulation, the MSS-31 cells formed a layer (Fig. 2B). The administration of MMT cell conditioned medium stimulated endothelial capillary-like morphogenesis (3.3+/-1.2 branching points per field of view) and this effect was exacerbated when medium was conditioned from Ets-1-overexpressing MMT cells (Fig. 2B) (5+/-1.7 branching points per field of view). By contrast, dominant negative DB mutant expression reduced MMT cell ability to induce endothelial cell morphogenesis, with the failure to trigger any tubular structure, further highlighting the critical role of Ets-1 in MMT cell angiogenic potential.

We then investigated angiogenic induction within the three-dimensional matrix gels under serum-free conditions, in order to remove the contribution of growth factors and proteases present in the serum. Endothelial cells embedded alone in these 3D matrix gels failed to form any cellular structure. The administration of MMT cell conditioned medium induced the alignment of elongated MSS-31 cells (with a mean structure length of $161 \mu \mathrm{m}$ ) (Fig. 2C). Ets-1 overexpression in MMT cells increased the mean length of MSS-31 structures $(251 \mu \mathrm{m}$; $\mathrm{P}=0.04)$, and $\mathrm{DB}$ expression did not alter it in a significant manner (142 $\mu \mathrm{m} ; \mathrm{P}=0.9)$ (Fig. 2C). Cell conditioned media were, however, not sufficient to trigger the formation of hollow lumen endothelial tubules within these gels.

As already explained in Fig. 1C, we set up 3D co-culture models of breast cancer and endothelial cells to further investigate the interactions between these cells. Endothelial cells, labeled with the intravital fluorescent $\mathrm{diO}$ probe prior to their seeding, were seeded upon 3D matrix gels containing MMT cancer cells. This model allowed us to follow endothelial capillary-like morphogenesis in the vicinity of breast cancer cells. The analysis of cell structures by epifluorescence revealed the implementation of an endothelial network with branched tubules in presence of MMT cells. Ets-1 overexpression in the MMT cells stimulated the architecture of endothelial tubules, whereas DB dominant negative mutant expression hindered their formation (Fig. 2D).

We also wished to determine which soluble factors may participate in this angiogenic induction. Ets-1 orchestrates matrix metalloproteinase (MMP) expression/activation by breast cancer cells (8) and MMPs are important for endothelial cell morphogenesis. In this study, we thus investigated whether MMPs produced by breast cancer cells could be recruited by endothelial cells. To do that, we analyzed the gelatinolytic (MMP-2/-9) activity of MMT conditioned media just after their administration to MSS-31 cells, or $24 \mathrm{~h}$ later. At both time-points, MMP-9 activity was mainly derived from MMT conditioned media, as there was almost no activity in the control MSS-31 cells condition (with the appropriate vehicle), and MMP-9 activity was associated with the Ets-1 status in MMT cells (Fig. 2E), in agreement with our previous findings (8). Nevertheless, MMP-9 activities in the media were decreased following a 24-h incubation with endothelial cells (right columns compared to left columns, Fig. 2E). MMP-2, which is mainly derived from the control medium, served as a loading control. In order to determine whether MMP-9 can be recruited by MSS-31 cells, we incubated the MSS-31 cells for a short period of time ( $5 \mathrm{~min}$ ) with MMT conditioned media, then thoroughly washed the cells 3 times. While the MSS-31 cell membrane extracts displayed no MMP-9 activity under the control conditions, those from the MSS-31 cells incubated with MMT cell conditioned media exhibited MMP-9 activities varying accordingly to MMT cell productions, whereas no MMP-2 activity was detected (Fig. 2F). Endothelial cells can thus recruit MMP-9 released by cancer cells.

Endothelial cells slightly decrease breast cancer cell proliferation and migration on plastic, but increase their invasive morphogenetic properties in matrix gels. After having ascertained MMT breast cancer cell angiogenic potential and having highlighted Ets-1 critical role in this process, we investigated the mechanisms through which endothelial cells can reciprocally modify breast cancer cell behaviour, as suggested in Fig. 1D.

We first assessed the ability of endothelial cell conditioned medium to influence MMT cell proliferation.. The proliferation of the three MMT cell sublines was reduced in presence of MSS-31 cell conditioned medium, although statistical significance was not reached for any of them (Fig. 3A). The chemotactic capacity of endothelial MSS-31 cells for MMT cells was then assessed with Transwell assays. We noted that the presence of MSS-31 cells induced a slight (but non-statistically significant) decrease in the migration of the 3 MMT cell sublines, although Ets-1-overexpressing cells still migrated to a greater extent than their counterparts (Fig. 3B). MSS-31 cell conditioned medium was also administered to wounded MMT cell cultures, and similarly slightly decreased their migration, as visualized $24 \mathrm{~h}$ later (Fig. 3C).

We further examined the response of the breast cancer cells to the endothelial cell presence in direct co-culture systems, as described above; i.e., with MSS-31 cells cultured upon matrix gels containing MMT cells. The branching morphogenesis of MMT cell sublines under control conditions, i.e., without endothelial cells above them, was characteristic of previously described features $(8,9)$. Ets-1 overexpression favoured individual invasion, while dominant negative DB expression induced the formation of thicker tubules when compared to MMT neo cells (Fig. 3D, top panels). Of note, MSS-31 culture above MMT-containing gels altered the morphogenesis of our 3 MMT cell sublines (Fig. 3D, bottom panels), with a decrease in the primary branch thickness (Fig. 3E) of the MMT neo and MMTDB cells. These findings demonstrate that endothelial cells per se can favour the expression of aggressive traits by cancer cells without providing them with any blood supply.

Ets-1 overexpression promotes breast cancer cell adhesion to endothelial cells, while decreasing their chemo-attractive potential for endothelial cells. Another key component of cancer cell interactions with endothelial cells in vivo is their ability to physically interact with the latter, which may physiologically affect their metastatic potential. Such interactions depend on two main parameters: Intercellular adhesion and chemoattraction. 

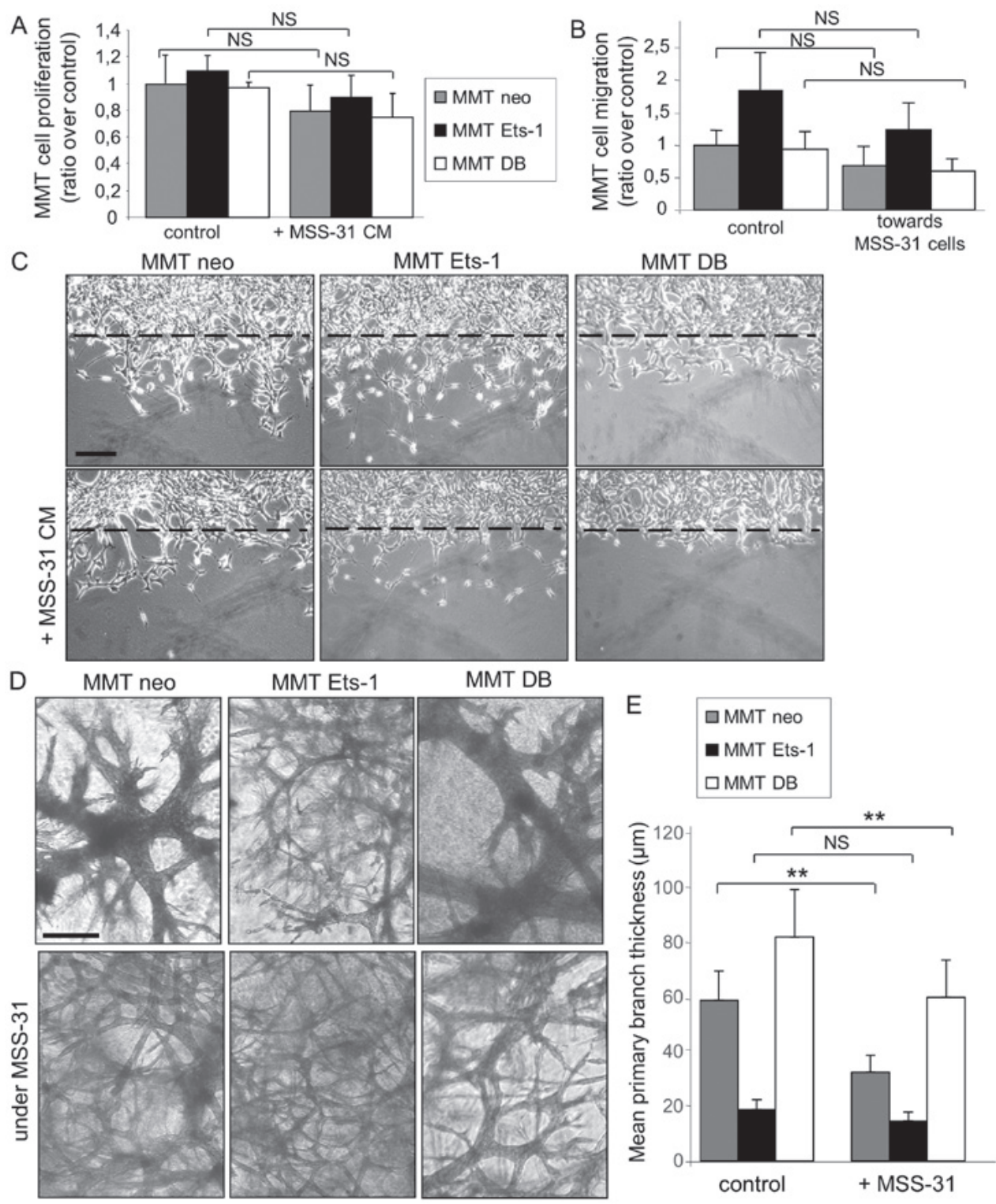

Figure 3. Endothelial cells slightly decrease breast cancer cell proliferation and migration on plastic, but increase their invasive morphogenetic properties in matrix gels. (A) MMT cell proliferation in response to the replacement of 30\% culture medium by MSS-31 cell conditioned media, or their culture medium as a control, was measured after a 3-day culture. Values are representative of 3 independent experiments. (B) MMT cells were seeded upon Transwell ${ }^{\circledR}$ inserts, and cultured in wells where MSS-31 endothelial cells (or no cells in the control condition) had been previously seeded. Values are means of 3 independent experiments. (C) MMT subconfluent cultures were wounded and incubated with MSS-31 cell conditioned media or their culture medium as a control. Images were acquired after a 24-h period of incubation, and show that Ets-1-driven MMT cell migration within the wound is decreased by endothelial cell conditioned media. (D) MMT cells were cultured within three-dimensional matrix gels, above which MSS-31 cells were deposited (bottom lane) or not (upper lane). Images were acquired following a 1-week culture and evidence endothelial cell-driven stimulation of cancer cell invasive branching morphogenesis. (E) Quantification of primary branch thickness was performed with ImageJ software and the results are plotted in the displayed graph. ${ }^{* *} \mathrm{P}<0.01$; NS, non-significant. Scale bars, $100 \mu \mathrm{m}$.

To evaluate whether Ets-1 regulates the processes of adhesion between cancer and endothelial cells, we examined whether the modulation of Ets-1 in cancer cells can alter their adherence to endothelial cells. MMT cell sublines were fluorescently labeled prior to their seeding on a confluent MSS-31 cell monolayer. Following $30 \mathrm{~min}$ of incubation, non-adherent cells were removed by 3 washes and epifluorescence analysis was performed to quantify the number of cancer cells attached to the endothelial layer. Of note, there were $41.2 \%(\mathrm{P}=0.04)$ more MMT Ets- 1 cells adherent to endothelial cells, and $24.8 \%(\mathrm{P}=0.056)$ less MMT DB cells adherent when compared with the MMT neo cells (Fig. 4A). We found that Ets-1 overexpression favored VE-cadherin expression in the MMT cells and DB mutant decreased it (Fig. 4B), highlighting a potential factor involved in these heterotypic interactions.

Physical interactions between cancer and endothelial cells also depend on their probability to meet each other. We previously reported that Ets-1 favored breast cancer cell migration (8), and we wished to determine whether it can also affect endothelial cell recruitment. For this purpose, we co-cultured the MSS-31 cells and MMT cells in Boyden chambers. Cancer cells were grown at the bottom of the wells, and endothelial cells were seeded upon inserts in these 

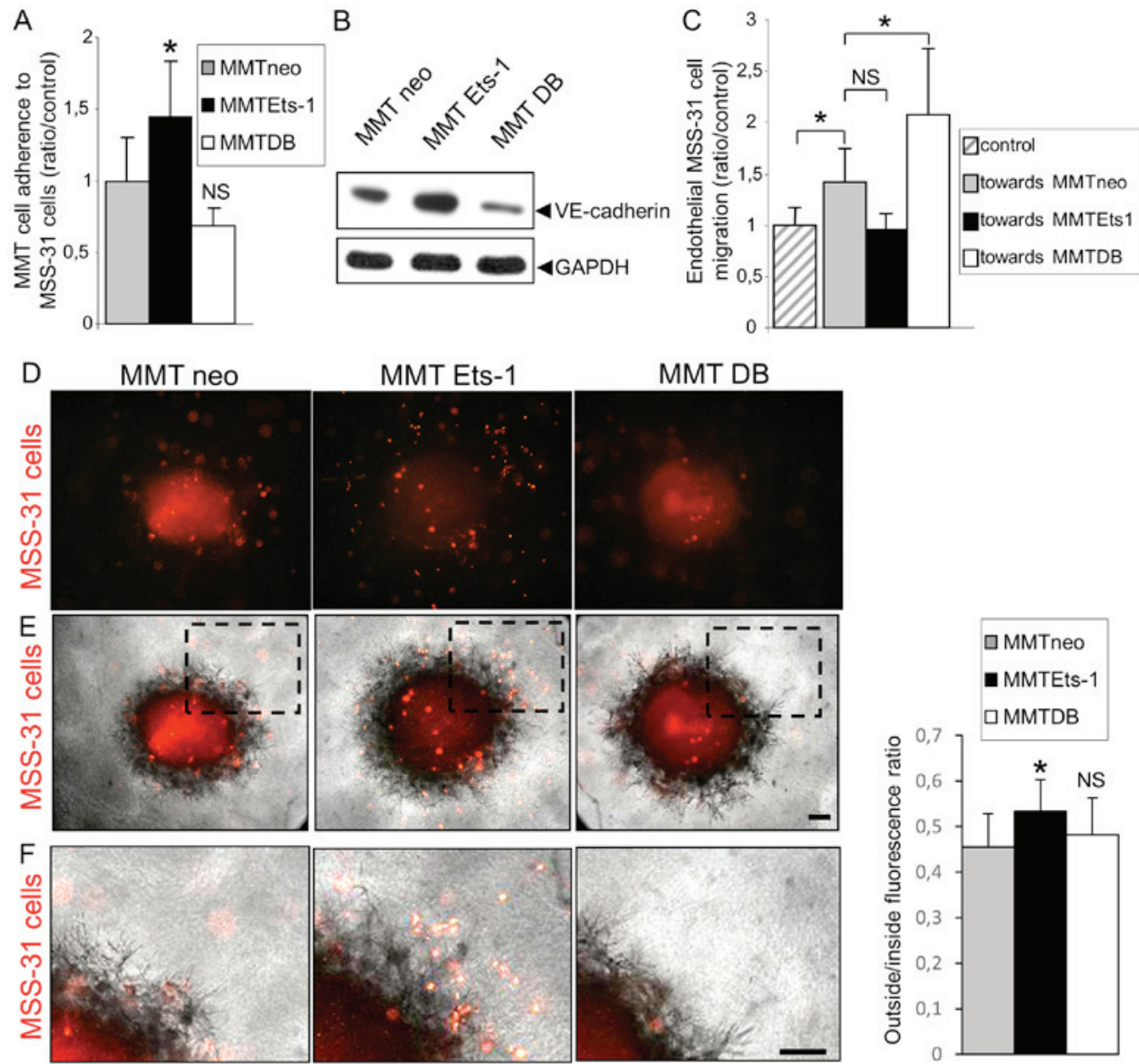

Figure 4. Ets-1 overexpression promotes breast cancer cell adherence to endothelial cells, but decreases their chemoattractive potential for endothelial cells. (A) Breast cancer cell adhesion to an endothelial cell layer was assessed $30 \mathrm{~min}$ after the addition of fluorescently-labelled MMT cell suspensions upon confluent monolayers of MSS-31 cells, and is increased in an Ets-1-dependent manner. Values are means of 3 independent experiments; ${ }^{*} \mathrm{P}<0.05$; NS, non-significant. (B) Immunoblotting was performed with MMT cell lysates and reveals the presence of VE-cadherin and the modulation of its expression by Ets-1. GAPDH was used as a loading control. (C) MSS-31 cells were seeded upon Transwell ${ }^{\circledast}$ inserts, and cultured in wells where MMT cells (or no cells in the control condition) had been previously seeded. Values are means of 3 independent experiments; "P<0.05; NS, non-significant. (D-F). Ex vivo MMT tumor fragments were deposited upon 3D matrix gels containing homogenously scattered diI-labeled MSS-31 cells. Endothelial cell (red fluorescence) recruitment by tumor fragments was assessed by (D) epifluorescence following a 3-day culture. " $\mathrm{P}<0.05$; NS, non-significant. A merge of the epifluorescent and phase contrast images is shown in (E). Dotted rectangles in (E) are magnified in (F). Scale bars, $50 \mu \mathrm{m}$.

wells. The presence of MMT neo cells increased MSS-31 cell migration by $35.2 \%(\mathrm{P}=0.03)$ when compared to the control condition without underlying cancer cells (Fig. 4C). Unexpectedly, Ets-1 overexpression in MMT cells led to a decrease in MSS-31 cell migration by $28.5 \%$ ( $\mathrm{P}=0.18$; NS), while DB mutant expression tended to increase it $(+35.8 \%$; $\mathrm{P}=0.04$ ), in comparison with the presence of MMT neo cells. We also evaluated the ability of ex vivo MMT tumor fragments retrieved from in vivo grafts in mice to recruit endothelial cells. These fragments were dropped on 3D matrix gels containing fluorescently labeled and homogenously scattered MSS-31 endothelial cells. MSS-31 cell distribution in these gels was followed over time by epifluorescence. Following a 3-day culture, control MMT neo and MMT DB fragments had recruited most endothelial cells in their core or their vicinity, whereas endothelial cells were still scattered around MMT Ets-1 tumor fragments (Fig. 4D and E, and enlargements in Fig. 4F). Fluorescence distribution was quantified inside and outside the fragment zone, and confirmed that endothelial cells were less recruited by MMT Ets-1 fragments (outside/ inside ratio of $53.4 \%$ vs. $45.5 \%$ for MMT neo, $\mathrm{P}=0.02$, and $48.2 \%$ for MMT DB, $\mathrm{P}=0.85$, NS when compared to MMT neo).

Ets-1 qualitatively alters MMT cell tumor vascularization in vivo. In order to understand the in vivo relevance of our afore-mentioned in vitro observations, we investigated tumor angiogenesis induction in MMT cell tumor xenografts using nude mice.

MMT cells subcutaneously injected with growth factor reduced Matrigel ${ }^{\mathrm{TM}}$ gave rise to tumors, whose angiogenesis was analyzed 10 days later. Mice were intravenously injected with FITC-dextran to visualize blood vessels and then euthanized and dissected to unveil the tumors from the covering skin. All MMT tumors were perfused with the systemic circulation independently of Ets-1 modulation (Fig. 5A). These experiments revealed that although Ets-1 overexpression hindered isolated endothelial cell recruitment in vitro, it did 

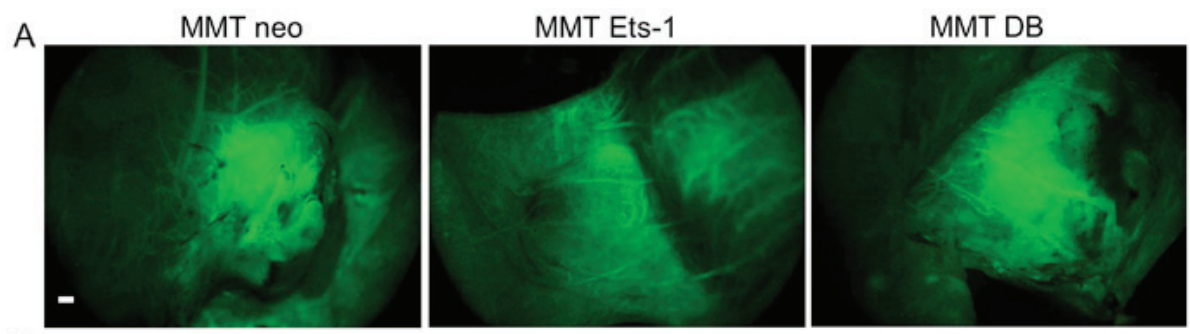

B
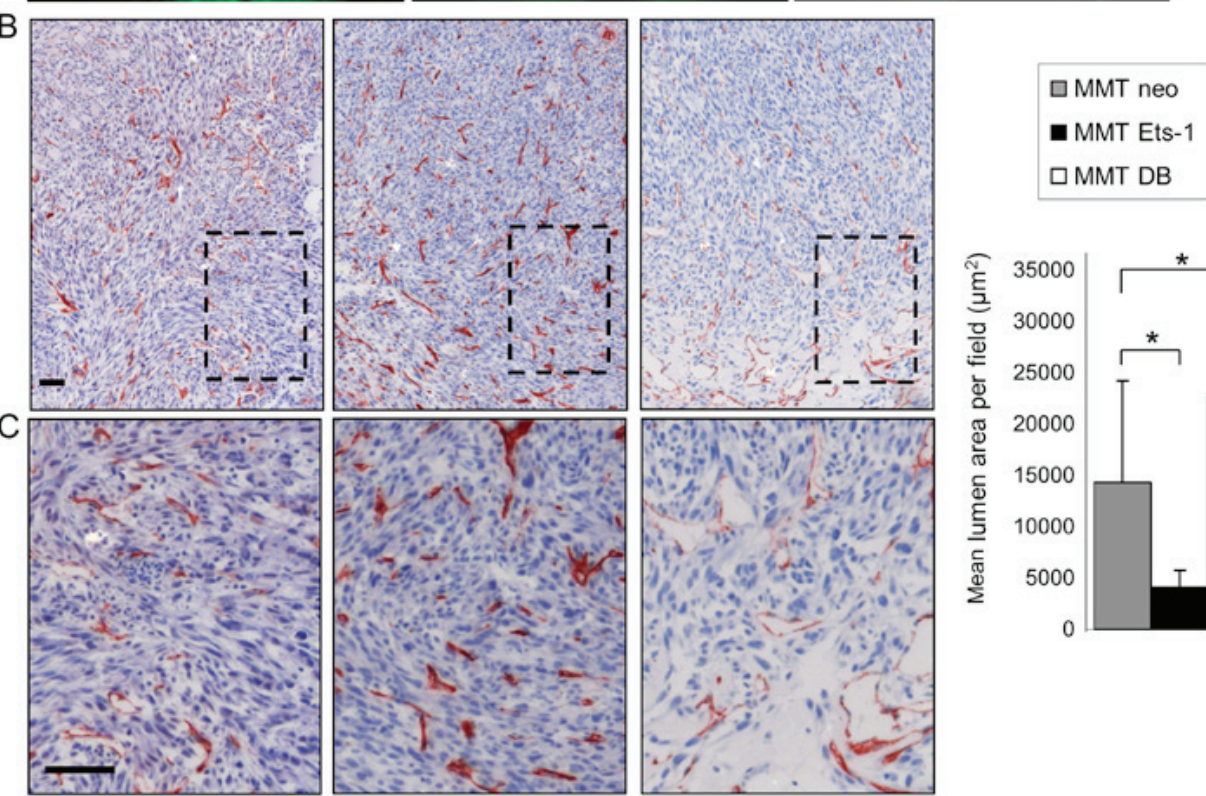

D

MMT neo

MMT Ets-1

MMT DB

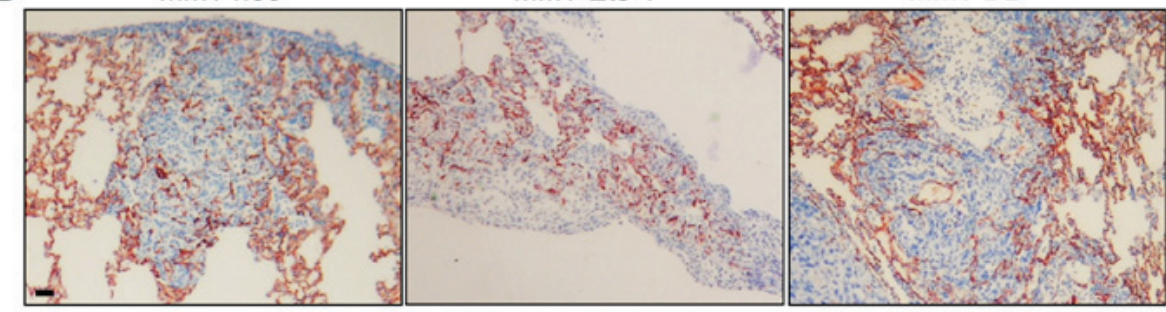

Figure 5. Ets-1 affects MMT cell tumor vascularization in vivo. (A) The external vasculature of MMT tumors that developed from subcutaneous injections of our different MMT cell sublines in nude mice was visualized after FITC-dextran injection to mice. Analysis by epifluorescence revealed that MMT tumors had all recruited and developed an important external vascular network. Scale bar, $1 \mathrm{~mm}$. (B and C) Immunohistochemical analysis of tumor angiogenesis with the endothelial marker, CD34. Dotted rectangles in (B) are magnified in (C). While MMT Ets-1 tumors display numerous capillaries, MMT DB tumors present larger vessels, particularly located at the tumor periphery. Tissues were counterstained with Mayer's hematoxylin. Cumulative capillary lumen surface was measured, and the mean area \pm SD was plotted; " $P<0.05$. (D) Lung metastases that developed after the intravenous injection of MMT cell sublines in nude mice were analysed 12 days after the injection. CD34 immunohistochemistry highlights blood vessel presence in the lung parenchyma and in metastases. Tissues were counterstained with Mayer's hematoxylin. Scale bars, $100 \mu \mathrm{m}$.

not impair the co-option in vivo by the tumor of surrounding blood vessels and its perfusion.

The tumors were then processed for immunostaining with a CD34 endothelial marker, which highlighted the presence of both large vessels and capillaries. This staining evidenced Ets-1-driven differences in the tumor vasculature features. While Ets-1 overexpression favored the presence of numerous capillaries in MMT tumors, DB mutant expressing tumors displayed only few intratumoral capillaries, but instead presented bigger vessels, particularly at the tumor periphery (Fig. 5B and C). This effect was quantified by measuring the capillary lumen areas on the tumor sections. The MMT neo tumor sections displayed a mean cumulative lumen surface of $14,301 \mu \mathrm{m}^{2}$ per field of view; Ets-1 overexpression by MMT tumors decreased this value to $4,075 \mu \mathrm{m}^{2}(\mathrm{P}=0.03)$, whereas the dominant negative increased it to $22,685 \mu \mathrm{m}^{2}$ $(\mathrm{P}=0.048)$ (Fig. 5B).

Since interactions between tumors and blood vessels play a key role in the metastatic process, we wished to determine whether the Ets-1-mediated modulation of these interactions results in the modification of the tumor metastatic potential. The rapid growth of MMT subcutaneous tumors however, prevented the observation of metastasis when mice were dissected. To circumvent this issue, we directly injected MMT cells into the tail vein of nude mice, which is a classical experimental metastasis assay. In this experimental frame, Ets-1 or its dominant negative mutant overexpression did not alter the number of lung metastases nor their vascularization, probably derived from the lung dense capillary network (Fig. 5D). MMT cell tropism for the lung, which is a highly vascularised organ, 
was not favourable to understand a putative angiogenic modulation by Ets-1 following cancer cell dissemination. These data merely indicate that Ets-1 modulation does not affect cancer cell entrapment in the lung capillaries.

\section{Discussion}

Cancer cell invasion and sprouting angiogenesis share some common molecular circuitry. Since Ets-1 orchestrates breast cancer cell invasion via different soluble factors, we reasoned that it may affect their angiogenic potential, which proved to be the case. Among such soluble factors, tumor-secreted MMP-9 has been reported to induce angiogenesis (16). In this study, we demonstrate that endothelial cells actually recruit cancer cell-derived MMP-9, and that the amount of recruited MMP-9 depends on Ets-1 expression/activity in cancer cells. Future experiments are warranted to unveil the importance of this mechanism in endothelial cell morphogenesis. We also demonstrate that Ets-1 overexpression in cancer cells promotes their adhesion to endothelial cells. VE-cadherin classically links adjacent endothelial cells, but can be expressed by aggressive cancer cells in what is known as vasculogenic mimicry (17). Ets-1 has been proposed to drive together with HIF-2 $\alpha$ the expression of the VE-cadherin gene by tumor cells (18). We actually found that Ets-1 favored VE-cadherin expression in MMT cells. In 3D co-culture models, we observed hybrid tubules made of cancer and endothelial cells. However, the rapid loss of cancer cell fluorescent labeling impaired us to quantify these structures and to investigate the putative impact of Ets-1 on vascular mimicry. With regard to cell adhesion, Ets-1 also controls $\beta 3$ subunit integrin expression (8), which can contribute to the adhesion of cancer cells to endothelial cells (19). To sum up, Ets-1 controls the expression of many molecules associated with adhesion and invasion. Therefore, it probably regulates angiogenic interactions via this network but not via a single molecule. Identifying the contribution of these individual actors and their combinations may be complex, since each actor can influence the others (e.g., MMPs can release or activate growth factors, which in turn control MMP expression...). Moreover, both cell partners can express the same molecules and reciprocally influence their secretion, further complicating such a type of study. In this study, we chose to rather focus on the phenotypic results of cellular interactions and dialog.

Importantly, this study highlights the reciprocal communication existing between cancer and endothelial cells. The in vitro signals elicited by endothelial cells demonstrate that these cells are far from being 'inert' cells whose only role is to form blood vessels in order to supply blood. This stands in agreement with other studies showing the importance of angiocrine factors in both normal and tumor settings. For example, it has been shown that during embryogenesis, primitive endothelial cells can control the developing endoderm (20) and that vessels contribute to pancreatic differentiation via inductive interactions (21).

In the field of tumorigenesis, much emphasis has been placed on the nutrient and oxygen supply since tumor angiogenesis was highlighted by Judah Folkman (22). The production by endothelial cells of paracrine factors potentially acting on tumor cells is yet a not so recent concept (23), but it has received little attention in the decade following its discovery. To the best of our knowledge, few studies have focused on reciprocal paracrine interactions between cancer and endothelial cells in co-culture. Bi-directional interactions have been reported in vitro between prostate cancer cells and endothelial cells (24). The induction by cancer cells of an autocrine endothelial activation was also evidenced in another co-culture model (25). With regard to in vivo relevance, it has beem described that endothelial cells can promote the early survival of tumor cells injected in mice, in a paracrine manner, prior to the onset of angiogenesis (26). More recently, it was shown that endothelial cords promote experimental melanoma growth in mice prior to their blood perfusion (27). Our in vitro data support this angiocrine notion and suggest that breast cancer cells may also be sensitive to such signals elicited by their endothelial partner in the tumor microenvironment.

In this study, we demonstrate that Ets-1 qualitatively controls tumor angiogenesis, rather than being a mere on/off switch. It is interesting to note that MMT DB tumors display larger blood vessels, particularly on their periphery. It is unlikely that such a vasculature is efficient enough to satisfy tumors with their needs, as evidenced by frequent necrotic areas. Conversely, Ets-1 expression in MMT tumors rather favors the formation of capillaries in the whole tumor. This network may sustain the release of angiocrine factors by activated endothelium, but is probably not a better guarantee of perfusion since tumor capillaries are often permeant and lack organization. These opposite angiogenic features may be reconciled in a same natural tumor if we consider that Ets-1 overexpression is classically not widespread in human breast tumors, but is rather induced in the tumor-stroma interaction zone (28). While the tumor bulk may recruit blood vessels, Ets-1-overexpressing cells can disseminate owing to their exacerbated motility capacities, which confer to them a high probability to meet partners in the nearby stroma. Moreover, their higher capacity to adhere to endothelial cells may help them to find a way in the systemic circulation via intravasation. Since the experimental metastasis assay used bypasses the early steps in the metastatic process, it does not help to elucidate whether Ets-1 putatively favors intravasation via pro-migratory and pro-adhesive activities. In the future, more sophisticated systems with for example, the surgical removal of the primary tumor, may help to highlight and unveil Ets-1 role in these early steps in vivo.

Of note, it has recently been shown that epithelial-tomesenchymal transition (EMT) phenotypes in tumor cells are associated with the release of soluble factors able to induce angiogenesis and recruit myeloid cells (29). In that study, Snail transcription factor was shown to mediate the induction of such factors. Targeting transcription factors such as Ets-1, Twist or Snail may thus represent a therapeutic approach to limit at the same time invasion and microenvironment activation including angiogenesis.

Many improvements have been achieved over the past years in understanding cancer angiogenesis, and have led to the development of promising therapeutic strategies for breast cancer patients (30). Nevertheless, several clinical trials with anti-angiogenic compounds have failed due to the adaptation of tumors, prompting researchers to obtain more insight into the tumor vasculature and propose new therapies (3). Vascular 
mimicry is associated with metastasis (31) and represents a clinical issue since it is a priori refractory to anti-angiogenic compounds. Identification of compounds blocking this process should be useful to fight cancers. We have also illustrated the role that angiocrine factors play in tumor development. In brief, a better understanding of angiogenesis modulation by tumors and of the complex interactions between cancer and endothelial cells may help us to tackle more efficiently this cancer hallmark and may lead to the development of effective treatment strategies for patients. Many opportunities are open and at the same time, much has still to be done to decipher the underlying mechanisms.

\section{Acknowledgements}

The authors are particularly grateful to Dr Fabrice Soncin and all members of our laboratories for providing tools, helpful discussions and comments.

\section{Funding}

This study was supported by CNRS, Association pour la Recherche contre le Cancer (ARC), Ligue Nationale contre le Cancer and Société Française du Cancer (SFC). AF was supported by Ministère de l'Education Nationale, Ligue Nationale contre le Cancer and Région Hauts de France (CPER Photonics 4 Society and 1-PRIMER).

\section{Availability of data and materials}

Data sharing is not applicable to this article, as no datasets were generated or analyzed during the current study.

\section{Authors' contributions}

AF, XD and AP conceived the study. AF and AP designed experiments. AF, CV, NW and AP performed experiments. $\mathrm{AF}, \mathrm{CV}, \mathrm{NW}, \mathrm{LH}, \mathrm{XD}$ and AP analysed and interpreted the data. AF, LH and AP wrote the manuscript. All authors have read and approved the final manuscript.

\section{Ethics approval and consent to participate}

In vivo experiments were performed according to approved institutional guidelines. Specific authorization no. 59-00994 was granted by the institutional veterinary authorities.

\section{Patient consent for publication}

Not applicable.

\section{Competing interests}

The authors declare that they have no competing interests.

\section{References}

1. Ferlay J, Soerjomataram I, Dikshit R, Eser S, Mathers C Rebelo M, Parkin DM, Forman D and Bray F: Cancer incidence and mortality worldwide: Sources, methods and major patterns in GLOBOCAN 2012. Int J Cancer 136: E359-E386, 2015.
2. Hansen S, Grabau DA, Sørensen FB, Bak M, Vach W and Rose C: The prognostic value of angiogenesis by Chalkley counting in a confirmatory study design on 836 breast cancer patients. Clin Cancer Res 6: 139-146, 2000

3. Carmeliet P and Jain RK: Molecular mechanisms and clinical applications of angiogenesis. Nature 473: 298-307, 2011.

4. Aalders KC, Tryfonidis K, Senkus E and Cardoso F: Antiangiogenic treatment in breast cancer: Facts, successes, failures and future perspectives. Cancer Treat Rev 53: 98-110, 2017.

5. Sivridis E, Giatromanolaki A and Koukourakis MI: The vascular network of tumours - what is it not for? J Pathol 201: 173-180, 2003.

6. Span PN, Manders P, Heuvel JJ, Thomas CMG, Bosch RR, Beex LVAM and Sweep CGJ: Expression of the transcription factor Ets-1 is an independent prognostic marker for relapse-free survival in breast cancer. Oncogene 21: 8506-8509, 2002.

7. Kola I, Brookes S, Green AR, Garber R, Tymms M, Papas TS and Seth A: The Ets1 transcription factor is widely expressed during murine embryo development and is associated with mesodermal cells involved in morphogenetic processes such as organ formation. Proc Natl Acad Sci USA 90: 7588-7592, 1993.

8. Furlan A, Vercamer C, Desbiens X and Pourtier A: Ets-1 triggers and orchestrates the malignant phenotype of mammary cancer cells within their matrix environment. J Cell Physiol 215: 782-793, 2008.

9. Furlan A, Vercamer C, Bouali F, Damour I, Chotteau-Lelievre A, Wernert N, Desbiens X and Pourtier A: Ets-1 controls breast cancer cell balance between invasion and growth. Int J Cancer 135: 2317-2328, 2014.

10. Yamada KM and Cukierman E: Modeling tissue morphogenesis and cancer in 3D. Cell 130: 601-610, 2007.

11. Mattot V, Vercamer C, Soncin F, Calmels T, Huguet C, Fafeur V and Vandenbunder B: Constitutive expression of the DNA-binding domain of Ets1 increases endothelial cell adhesion and stimulates their organization into capillary-like structures. Oncogene 19: 762-772, 2000.

12. Yanai N, Satoh T and Obinata M: Endothelial cells create a hematopoietic inductive microenvironment preferential to erythropoiesis in the mouse spleen. Cell Struct Funct 16: 87-93, 1991.

13. Bradford MM: A rapid and sensitive method for the quantitation of microgram quantities of protein utilizing the principle of protein-dye binding. Anal Biochem 72: 248-254, 1976.

14. Pourtier-Manzanedo A, Vercamer C, Van Belle E, Mattot V, Mouquet $F$ and Vandenbunder B: Expression of an Ets-1 dominant-negative mutant perturbs normal and tumor angiogenesis in a mouse ear model. Oncogene 22: 1795-1806, 2003.

15. Arnaoutova I, George J, Kleinman HK and Benton G: The endothelial cell tube formation assay on basement membrane turns 20: State of the science and the art. Angiogenesis 12: 267-274, 2009.

16. Mira E, Lacalle RA, Buesa JM, de Buitrago GG, JiménezBaranda S, Gómez-Moutón C, Martínez-A C and Mañes S: Secreted MMP9 promotes angiogenesis more efficiently than constitutive active MMP9 bound to the tumor cell surface. J Cell Sci 117: 1847-1857, 2004.

17. Hendrix MJC, Seftor EA, Hess AR and Seftor REB: Vasculogenic mimicry and tumour-cell plasticity: Lessons from melanoma. Nat Rev Cancer 3: 411-421, 2003.

18. Le Bras A, Lionneton F, Mattot V, Lelièvre E, Caetano B, Spruyt N and Soncin F: HIF-2alpha specifically activates the VE-cadherin promoter independently of hypoxia and in synergy with Ets-1 through two essential ETS-binding sites. Oncogene 26: 7480-7489, 2007

19. Leroy-Dudal J, Demeilliers C, Gallet O, Pauthe E, Dutoit S, Agniel R, Gauduchon P and Carreiras F: Transmigration of human ovarian adenocarcinoma cells through endothelial extracellular matrix involves alphav integrins and the participation of MMP2. Int J Cancer 114: 531-543, 2005.

20. Bahary N and Zon LI: Development. Endothelium - chicken soup for the endoderm. Science 294: 530-531, 2001.

21. Lammert E, Cleaver O and Melton D: Induction of pancreatic differentiation by signals from blood vessels. Science 294: 564-567, 2001

22. Folkman J: Tumor angiogenesis: Therapeutic implications. N Engl J Med 285: 1182-1186, 1971.

23. Rak J, Filmus J and Kerbel RS: Reciprocal paracrine interactions between tumour cells and endothelial cells: The 'angiogenesis progression’ hypothesis. Eur J Cancer 32A: 2438-2450, 1996. 
24. Barrett JM, Mangold KA, Jilling T and Kaul KL: Bi-directional interactions of prostate cancer cells and bone marrow endothelial cells in three-dimensional culture. Prostate 64: 75-82, 2005.

25. Khodarev NN, Yu J, Labay E, Darga T, Brown CK, Mauceri HJ, Yassari R, Gupta N and Weichselbaum RR: Tumour-endothelium interactions in co-culture: Coordinated changes of gene expression profiles and phenotypic properties of endothelial cells. J Cell Sci 116: 1013-1022, 2003.

26. Shan S, Robson ND, Cao Y, Qiao T, Li CY, Kontos CD, GarciaBlanco $\mathrm{M}$ and Dewhirst MW: Responses of vascular endothelial cells to angiogenic signaling are important for tumor cell survival. FASEB J 18: 326-328, 2004

27. Zhao C, Zhang W, Zhao Y, Yang Y, Luo H, Ji G, Dong E, Deng H, Lin S, Wei Y, et al: Endothelial cords promote tumor initial growth prior to vascular function through a paracrine mechanism. Sci Rep 6: 19404, 2016.

28. Behrens P, Rothe M, Wellmann A, Krischler J and Wernert N: The Ets-1 transcription factor is up-regulated together with MMP 1 and MMP 9 in the stroma of pre-invasive breast cancer. J Pathol 194: 43-50, 2001.
29. Suarez-Carmona M, Bourcy M, Lesage J, Leroi N, Syne L, Blacher S, Hubert P, Erpicum C, Foidart JM, Delvenne P, et al: Soluble factors regulated by epithelial-mesenchymal transition mediate tumour angiogenesis and myeloid cell recruitment. J Pathol 236: 491-504, 2015.

30. Earl HM, Hiller L, Dunn JA, Blenkinsop C, Grybowicz L, Vallier AL, Abraham J, Thomas J, Provenzano E, HughesDavies L, et al; ARTemis Investigators: Efficacy of neoadjuvant bevacizumab added to docetaxel followed by fluorouracil, epirubicin, and cyclophosphamide, for women with HER2negative early breast cancer (ARTemis): An open-label, randomised, phase 3 trial. Lancet Oncol 16: 656-666, 2015.

31. Wagenblast E, Soto M, Gutiérrez-Ángel S, Hartl CA, Gable AL, Maceli AR, Erard N, Williams AM, Kim SY, Dickopf S, et al: A model of breast cancer heterogeneity reveals vascular mimicry as a driver of metastasis. Nature 520: 358-362, 2015.

(c) (9) This work is licensed under a Creative Commons

cc) Attribution-NonCommercial-NoDerivatives 4.0 International (CC BY-NC-ND 4.0) License. 